\title{
The Effect of Spike and Gap Deconvolution on 2D Land Seismic Data
}

\author{
Hassan Z. Ali, Hassan A. Thabit \\ Minstry of Oil, Oil Exploration Company, Baghdad, Iraq \\ Email: Hassan.zaid1989@yahoo.com, ha.thabit@yahoo.com
}

How to cite this paper: Ali, H.Z. and Thabit, H.A. (2018) The Effect of Spike and Gap Deconvolution on 2D Land Seismic Data. Open Access Library Journal, 5: e4594.

https://doi.org/10.4236/oalib.1104594

Received: April 11, 2018

Accepted: May 18, 2018

Published: May 21, 2018

Copyright $\odot 2018$ by authors and Open Access Library Inc.

This work is licensed under the Creative Commons Attribution International License (CC BY 4.0).

http://creativecommons.org/licenses/by/4.0/

\section{(c) (i) Open Access}

\begin{abstract}
In this paper we are performing Spiking and Gap deconvolution on land 2D data; we are applying on the shot and stack data with operator length (200 $\mathrm{ms}$ ), while in the gap deconvolution it is used different gap window (16.24 $\mathrm{ms})$. The quality of output data is better than input data, and there is homogeneity in the distribution of frequencies. Also the band width of frequencies is increasing and smoothing specialist in the case of spike deconvolution because it increases the temporal resolution so, it appears for us the inter beds between the layers and this is very important about the interpretation.
\end{abstract}

\section{Subject Areas}

Geophysics

\section{Keywords}

Spike Deconvoluion, Gap Deconvoluion, Seismic Data Processing and Land Seismic Data

\section{Introduction}

The definition of deconvolution is a filtering process that removes a wavelet from the recorded seismic trace [1] and is done by reversing the process of convolution [2]. The commonest ways that perform deconvolution, by designing a Wiener filter to transform one wavelet into another wavelet in a least-squares sense [3]. Deconvolution concerns itself with removing a part of the data which is convolutional. For instance, we know that a total seismic response consists of the convolution of the seismic source wavelet with the earth response, convolved with the response from the seismic detector, convolved with the seismic response from the recording system. If we consider only the seismic source signa- 
ture $s(t)$ and the impulse response of the earth $g(t)$, then the seismic signal can be written as shown in Equation (1) [4].

$$
x(t)=g(t) * s(t)
$$

\section{Optimum Wiener Filter}

It is filter designed to find the inverse filter to convert the basic seismic wave to any desired output the zero-delay spike $(1,0,0)$, that was considered when studying inverse and least-squares filters Rewrite Equation (2), which we solved to obtain the least-squares inverse filter, as follows [5]:

$$
\begin{gathered}
\left(\begin{array}{cc}
5 / 2 & -1 \\
-1 & 5 / 2
\end{array}\right)\left(\begin{array}{l}
a \\
b
\end{array}\right)=\left(\begin{array}{l}
2 \\
0
\end{array}\right) \\
2\left(\begin{array}{cc}
5 / 4 & -1 / 2 \\
-1 / 2 & 5 / 4
\end{array}\right)\left(\begin{array}{l}
a \\
b
\end{array}\right)=\left(\begin{array}{l}
2 \\
0
\end{array}\right)
\end{gathered}
$$

Divide both sides by 2 to obtain:

$$
\left(\begin{array}{cc}
5 / 4 & -1 / 2 \\
-1 / 2 & 5 / 4
\end{array}\right)\left(\begin{array}{l}
a \\
b
\end{array}\right)=\left(\begin{array}{l}
2 \\
0
\end{array}\right)
$$

The autocorrelation of the input wavelet $(1,-1 / 2)$ is shown in Table 1 Note that the autocorrelation lags are the same as the first column of the $2 \times 2$ matrix on the left side of Equation (3). Now compute the cross correlation of the desired output $(1,0,0)$ with the input wavelet $(1,-1 / 2)$ (Table 2$)$. The crosscorrelation lags are the same as the column Matrix on the right side of Equation (3). In general, the elements of the matrix on the left side of Equation (3) are the lags of the autocorrelation of the input wavelet, while the elements of the column matrix on the right side are the lags of the crosscorrelation of the desired output with the input wavelet, show Figure 1.

Now perform similar operations for wavelet $(-1 / 2,1)$. By rewriting the matrix Equation (2)-(19), we obtain:

$$
2\left(\begin{array}{cc}
5 / 4 & -1 / 2 \\
-1 / 2 & 5 / 4
\end{array}\right)\left(\begin{array}{l}
a \\
b
\end{array}\right)=\left(\begin{array}{c}
-1 \\
0
\end{array}\right)
$$

Divide both sides by 2 to obtain:

$$
\left(\begin{array}{cc}
5 / 4 & -1 / 2 \\
-1 / 2 & 5 / 4
\end{array}\right)\left(\begin{array}{l}
a \\
b
\end{array}\right)=\left(\begin{array}{c}
-1 \\
0
\end{array}\right)
$$

Matrix Equations (3) and (6) were used to derive the least-squares inverse filters. These filters then were applied to the input wavelets to compress them to

Table 1. Shows Autocorrelation lags of input wavelet $(1,-1 / 2)$ [5].

\begin{tabular}{ccc}
\hline Wavelet signature & Inverse filter & \\
\hline 1 & $-1 / 2$ & Output desired \\
1 & $-1 / 2$ & $5 / 4$ \\
& 1 & $-1 / 2$ \\
\hline
\end{tabular}


Table 2. Shows Cross correlation lags of desired output $(1,0,0)$ with input wavelet (1, $-1 / 2)[5]$.

\begin{tabular}{cccc}
\hline Wavelet signature & Inverse filter & \\
\hline 1 & 0 & 0 & Output desired \\
1 & $-1 / 2$ & $-1 / 2$ & 1 \\
& 1 & 0 \\
\hline
\end{tabular}

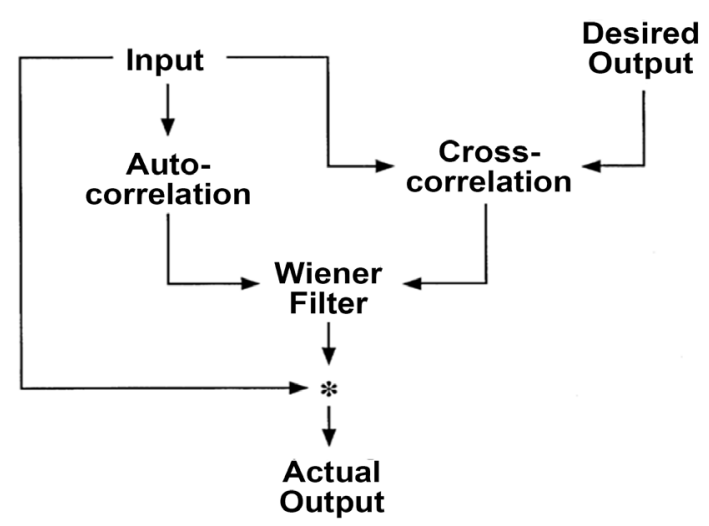

Figure 1. A flowshart for weiner filter design and application [5].

zero-lag spike. The matrices on the left in Equations (3) and (6) are made up of the autocorrelation lags of the input wavelets. Additionally, the column matrices on the right are made up of lags of the crosscorrelation of the desired output a zero lag spike, with the input wavelets. These observations were generalized by Wiener to derive filters that convert the input to any desired output [5].

\section{Algorithm Principle}

The spiking deconvolution in seismic data processing is routinely applied to compress the source wavelet included in the seismic traces to improve temporal resolution. The general form of the matrix equation for a filter of length $\mathrm{n}$ is represented in Equation (7), [6]:

$$
\left[\begin{array}{cccc}
r_{0} & r_{1} & r_{2} & r_{n-1} \\
r_{1} & r_{0} & r_{1} & r_{n-2} \\
r_{2} & r_{1} & r_{0} & r_{n-3} \\
r_{n-1} & r_{n-2} & r_{n-3} & r_{0}
\end{array}\right]\left[\begin{array}{c}
a_{0} \\
a_{1} \\
a_{2} \\
a_{n-1}
\end{array}\right]=\left[\begin{array}{c}
g_{0} \\
g_{1} \\
g_{2} \\
g_{n-1}
\end{array}\right]
$$

Here, $r_{i}, a_{p}$ and $g_{p} \quad i=0,1,2, \cdots n-1$ are the autocorrelation lags of the input wavelet, Winer coefficients, and the Crosscorrelation lags of the desired output with the input wavelet respectively. If the desired output is zero delay spike, it is call spiking deconvolution (Equation (8)):

$$
\left[\begin{array}{cccc}
r_{0} & r_{1} & r_{2} & r_{n-1} \\
r_{1} & r_{0} & r_{1} & r_{n-2} \\
r_{2} & r_{1} & r_{0} & r_{n-3} \\
r_{n-1} & r_{n-2} & r_{n-3} & r_{0}
\end{array}\right]\left[\begin{array}{c}
a_{0} \\
a_{1} \\
a_{2} \\
a_{n-1}
\end{array}\right]=\left[\begin{array}{l}
1 \\
0 \\
0 \\
0
\end{array}\right]
$$


Filter has the same form as the matrix in Equation (8). Therefore, spiking deconvolution is mathematically identical to least squares inverse filter. A distinction, however, is made in practice between the two types of filtering. The autocorrelation matrix on the left side of Equation (8) is computed from the input seismogram, in the case of spiking deconvolution (statistical deconvolution), whereas it is computed directly from the known source wavelet in case of least squares inverse filtering. If the input wavelet is not a minimum phase, spiking deconvolution cannot convert it to a perfect zero-lag spike. Although the amplitude spectrum is virtually flat, the phase spectrum of the output is not a minimum phase. The spiking deconvolution operator is the inverse of the minimum-phase equivalent of the input wavelet. This wavelet may or may not be minimum phase [7]. The predicative deconvolution desired output, a time advance from of input series suggests a predication processes. Given input $\mathrm{x}(t)$, we want to predict its value at some full time $(t+\alpha)$, where $\alpha$ is predication lag. Wiener show that the filter used to estimate $(x+\alpha)$ can be computed by using a special form of the matrix Equation (9):

$$
\left[\begin{array}{cccc}
r_{0} & r_{1} & r_{2} & r_{n-1} \\
r_{1} & r_{0} & r_{1} & r_{n-2} \\
r_{2} & r_{1} & r_{0} & r_{n-3} \\
r_{n-1} & r_{n-2} & r_{n-3} & r_{0}
\end{array}\right]\left[\begin{array}{c}
a_{0} \\
a_{1} \\
a_{2} \\
a_{n-1}
\end{array}\right]=\left[\begin{array}{c}
g_{0} \\
g_{1} \\
g_{2} \\
g_{n-1}
\end{array}\right]
$$

where Here $r_{p} a_{i}$ and $g_{i}, i=0,1,2 \cdots n, n-1$ are the autocorrelation lags of the input wavelet, the Wiener filter coefficients, and the cross correlation lags of the desired output with the input wavelet. Since the desired output $x(t+\alpha)$ is the time-advance version of the input $x(t)$, we need to specialize the right side of Equation (4) for the predication problem. Consider a Five-point input time series $x(t):(x 0, x 1, x 2, x 3, x 4)$, and set $\alpha=2$. The designed may be carried out using Equation (10) [7]:

$$
\left[\begin{array}{cccc}
r_{0} & r_{1} & r_{2} & r_{n-1} \\
r_{1} & r_{0} & r_{1} & r_{n-2} \\
r_{2} & r_{1} & r_{0} & r_{n-3} \\
r_{n-1} & r_{n-2} & r_{n-3} & r_{0}
\end{array}\right]\left[\begin{array}{c}
a_{0} \\
a_{1} \\
a_{2} \\
a_{n-1}
\end{array}\right]=\left[\begin{array}{c}
r_{a} \\
r_{a+1} \\
r_{a+2} \\
r_{a+n-1}
\end{array}\right]
$$

\section{Methodology}

The advantages of this method, we get clear data have more resolution and formatted to the migration method, so it is become ready to the interpretation. The deconvolution is the essential stage and it is consider important case in the seismic processing sequence because it is increase the temporal resolution and compressed the wavelet, in addition of decreases the noise compared with the signal. Deconvolution of seismic data can be performed in two types (spike and gap deconvolution) depending on the used source and the requirements of interpreter. Mostly, two techniques (spike and gap) used in the processing of data and the best results are selected to the production. In our work we showed the effective of (spike and gap) deconvolution on the shot domain free from any 
noise as shown in the Figure 2. The using system it is called Geovation (CGG company).

\section{Results}

This process is executed by following steps, firstly we are going to apply spike deconvolution on the shot data free from any noise with operator length (200 $\mathrm{ms}$ ), and second apply the predictive (gap) deconvolution and the using gap (16 $\mathrm{ms}$ ) with the same operator gap $200 \mathrm{~ms}$ also. So, from these results we show the difference between input shot data and spike deconvolution. Notice that there is enhancement in the temporal resolution after spike deconvolution apply and there is high clear in the hyperbola of shot data specialist in the initial of time about $600 \mathrm{~ms}$ as shown in Figure 3(a), Figure 3(b). these difference gives impression about the spectrums so, there is smoothing in the band width of frequencies better than spectrums without spike deconvolution applying, because of the last work compressed for wavelets like the wavelet before inner subsurface and It is re-frequencies that lost during subsurface layers so that, the bandwidth frequencies of wavelet increases low and high that it is led to enhance in the temporal resolution as shown in Figure 4(a), Figure 4(b). Figure 5(a), Figure 5(b) show the distributions of frequencies with applying of spike deconvolution and without apply it. So there is homogenous in the distribution of frequencies specialist in the initial time, it is mean high frequencies in the initial time of the shot data so, there is high resolution in this time. The wavelet reconverted to the normal case before interred the subsurface layers and there is re-frequncies to the wavelet compared with the other case without apply spike deconvolution. There is randomly in frequencies and attenuation because of the wavelet passed during the sequences of processes in the subsurface layers such as absorption refraction and dispersion. In the gap deconvolution there is preserve in the wavelet

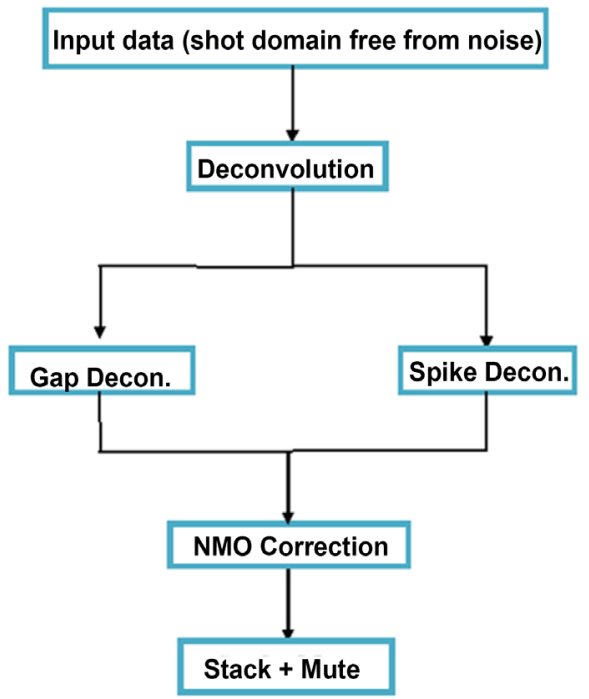

Figure 2. Shows the flow processing of gap and spike deconvolution. 


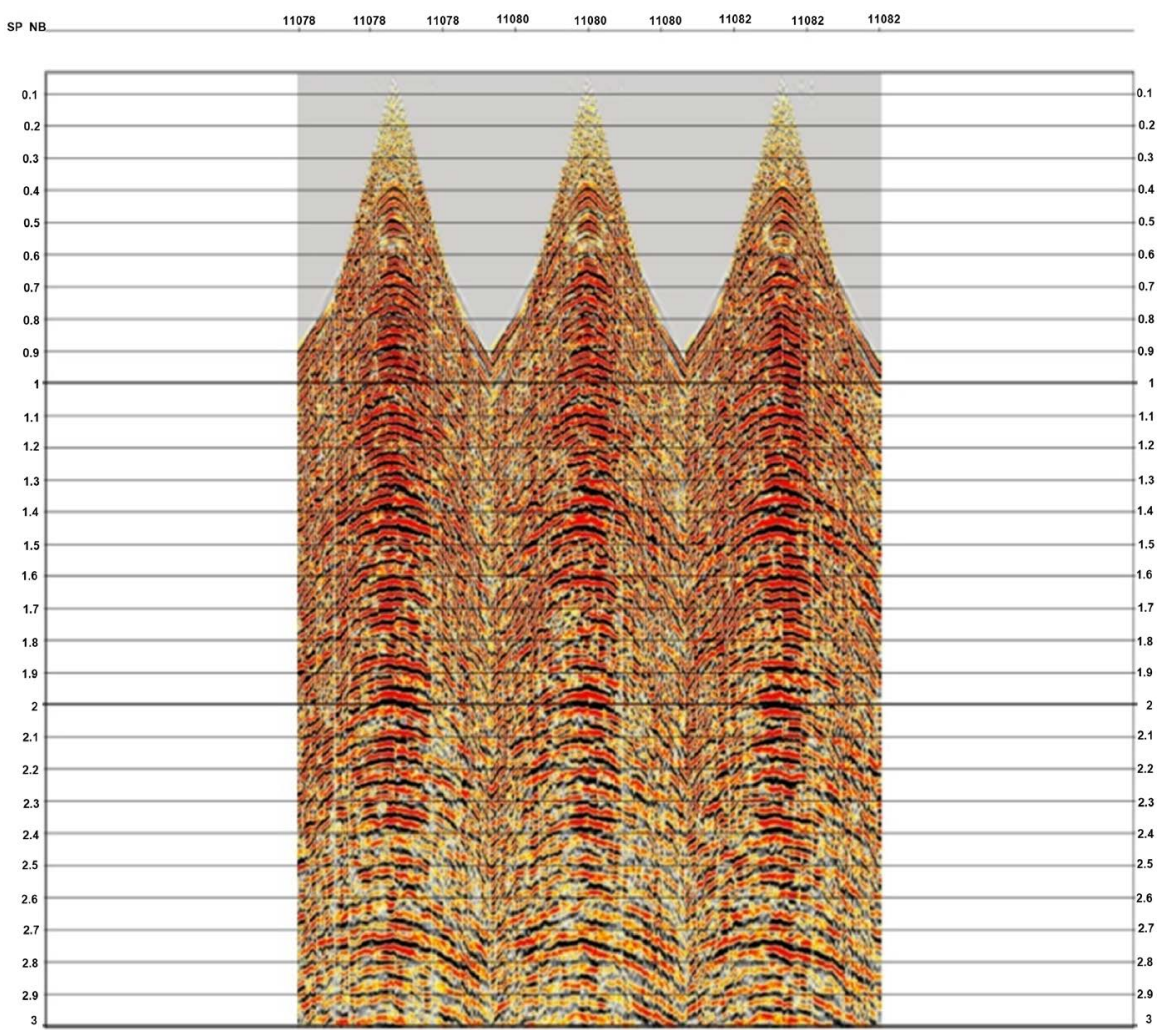

(a)

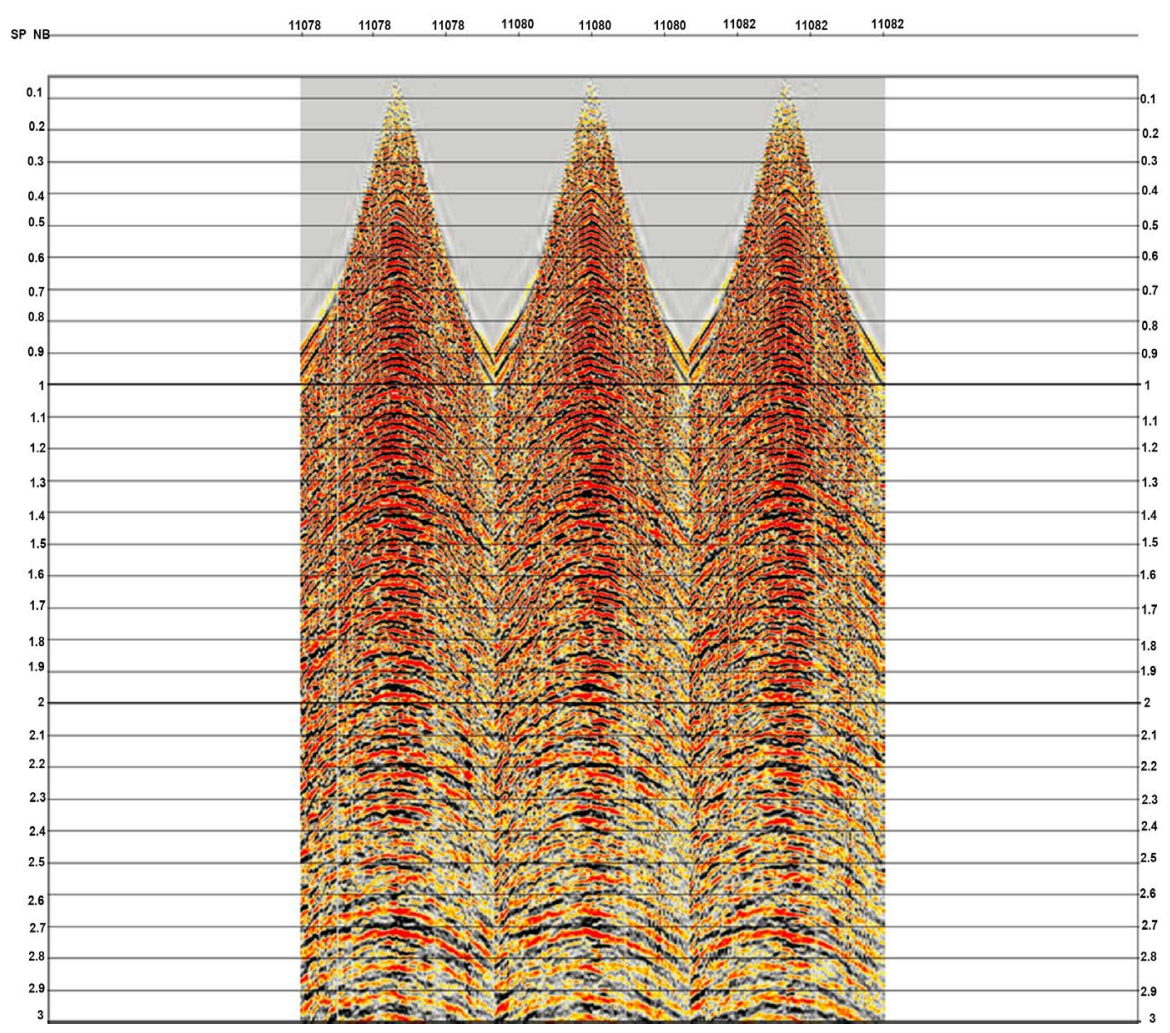

(b)

Figure 3. (a) Shot data free from noise before spike deconvolution; (b) Spike deconvolution with operator length $200 \mathrm{~ms}$. 


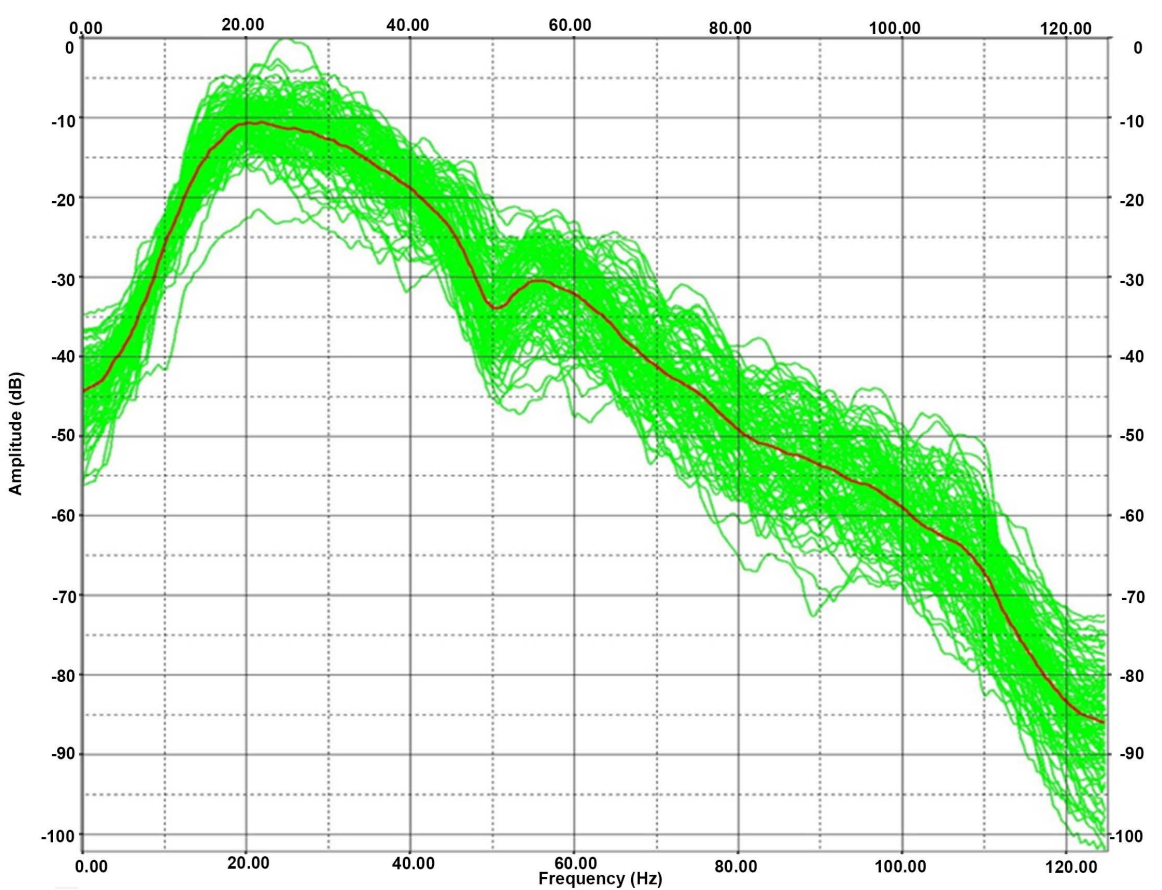

(a)

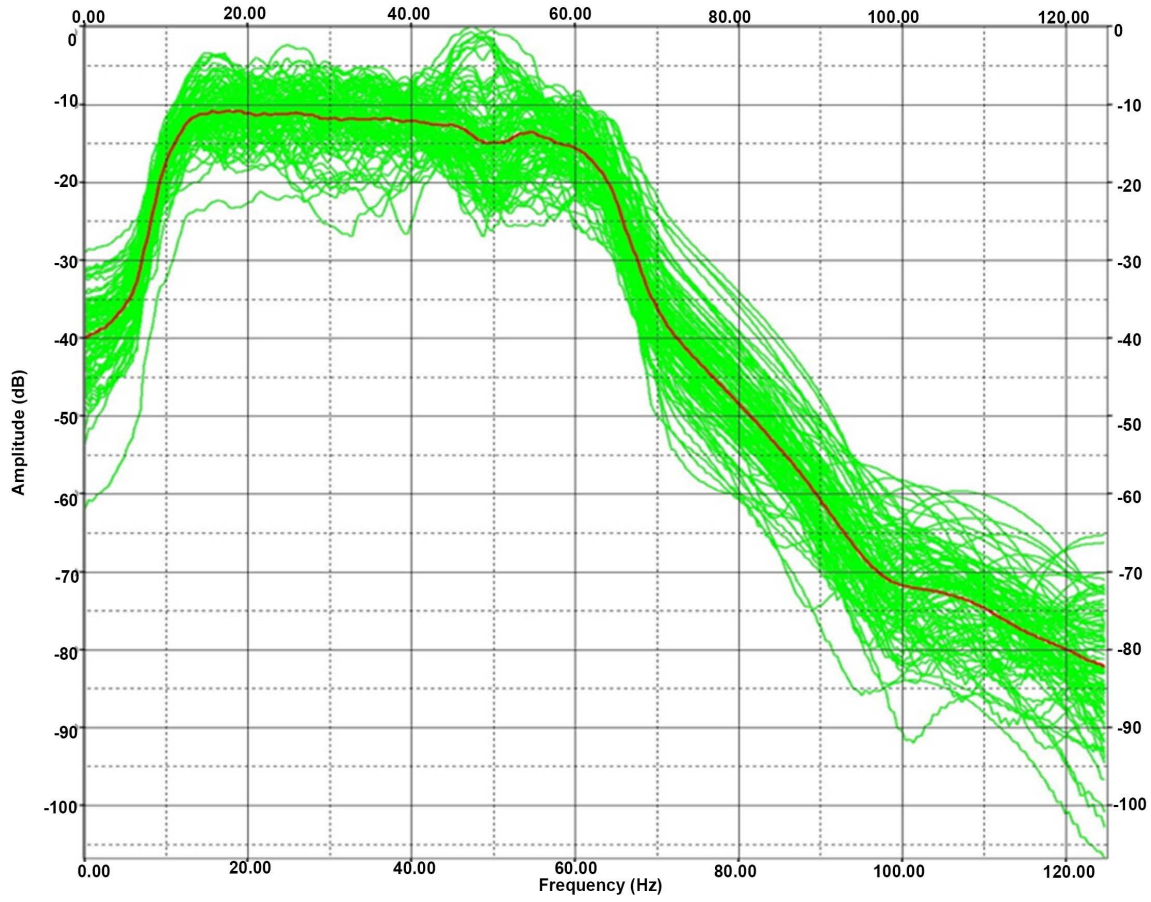

(b)

Figure 4. (a) Spectrum of band width frequencies without spike decinvolution; (b) Spectrum of band width frequencies with spike deconvolution.

shape and continuity in the reflection data but there is no resolution compared with the spike deconvolution. The signal to the nose ratio enhances and attenuates in the multiples reflections when the window of gap is decreases because of the decreasing of gap refer to the compressing of wavelet without sidesloops. Also 


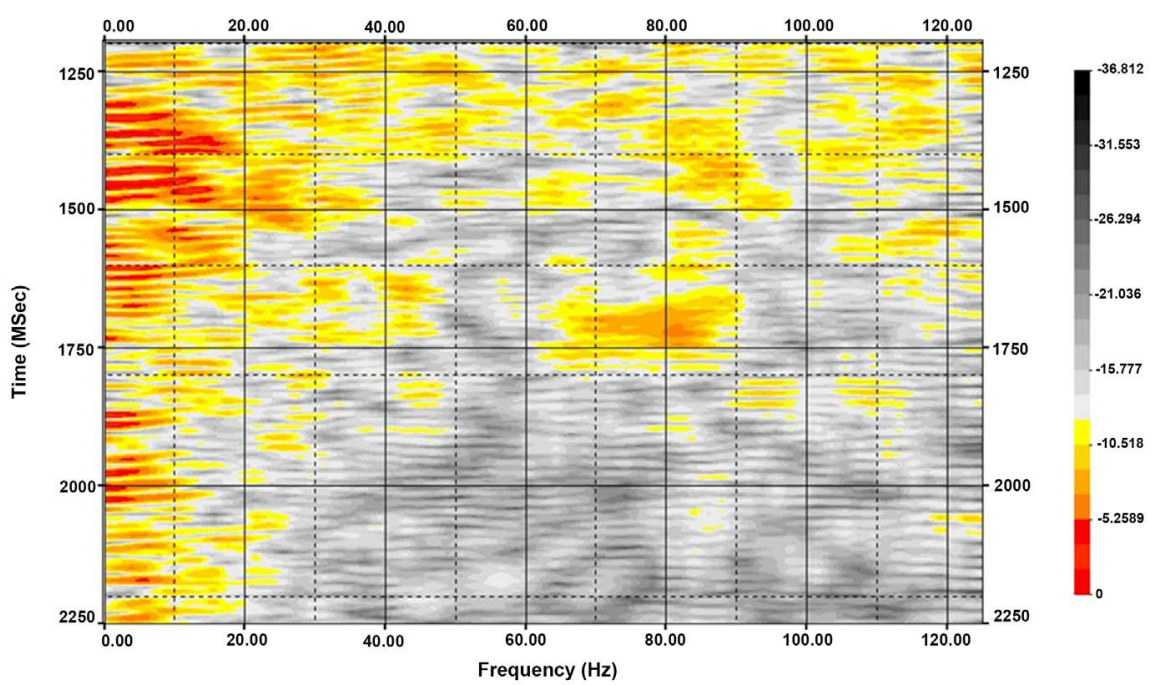

(a)

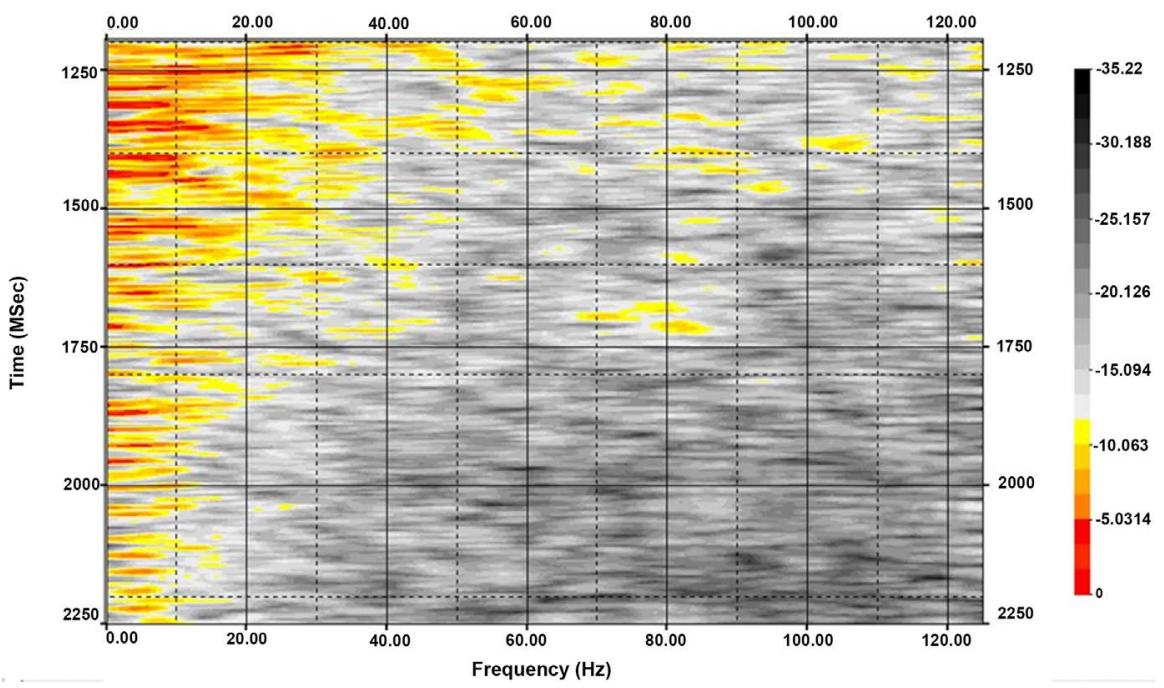

(b)

Figure 5. (a) Shows the distributions of frequencies without spike deconvolution; (b) Shows the distributions of frequencies with spike deconvolution.

the spectrum of frequencies is flatting as shown in Figure 6(a), Figure 6(b). The difference in the bandwidth of frequencies between the spike and gap deconvolution, In the case of spike deconvolution we notice the spectrum more flattening and the bandwidth is wider compared with the bandwidth of the gap deconvolution, as we said the spike deconvolution compressed the wavelet led to increase in the bandwidth frequencies, so the temporal resolution is increase as shown in Figure 4(b), Figure 6(b).

Figure $7(\mathrm{a})$, Figure $7(\mathrm{~b})$ shows comparing the stack data before and after deconvolution, there is enhancement in the seismic stack after deconvolution, flatting reflectors which appear specialist around initial time $750 \mathrm{~ms}$ and also 950 ms as shown in Figure 7(b). Additionally inter bed layers appeared because the temporal resolution. This improvement in the vertical resolution and enhanced 


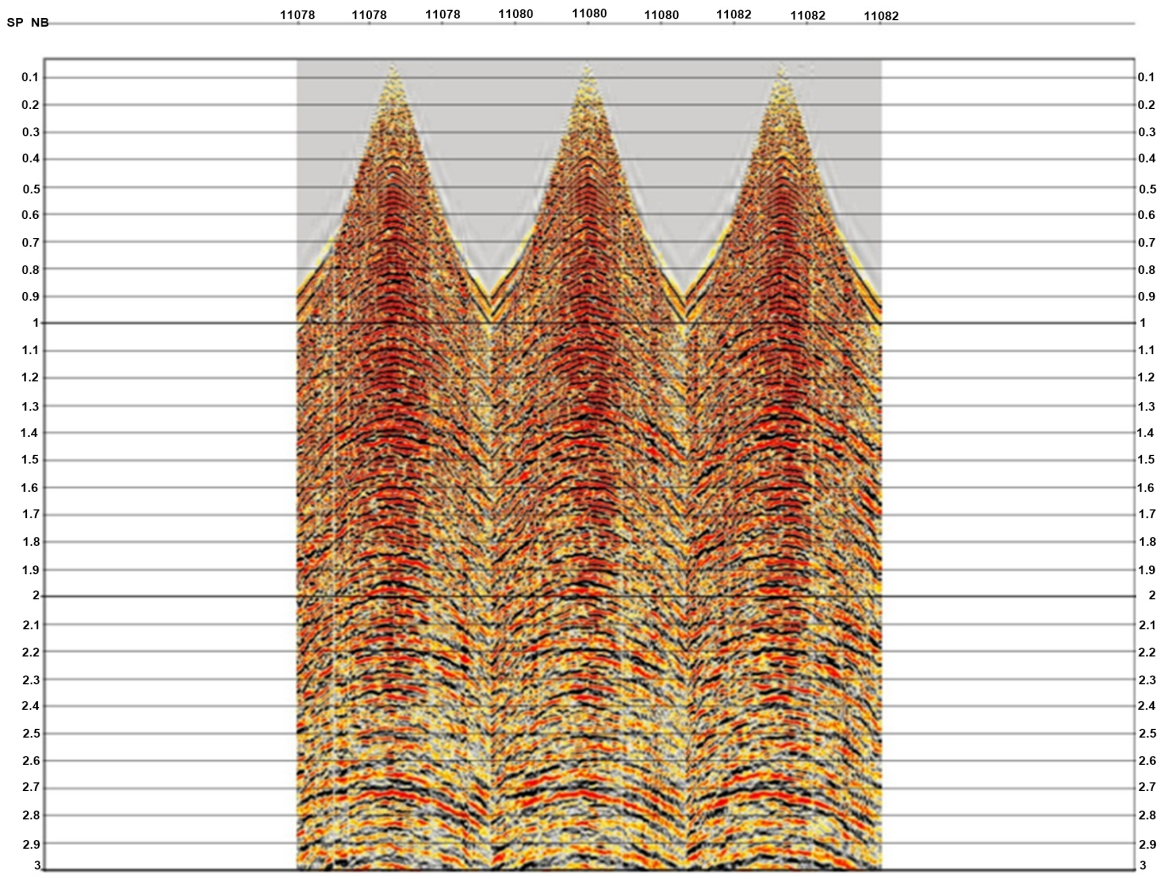

(a)

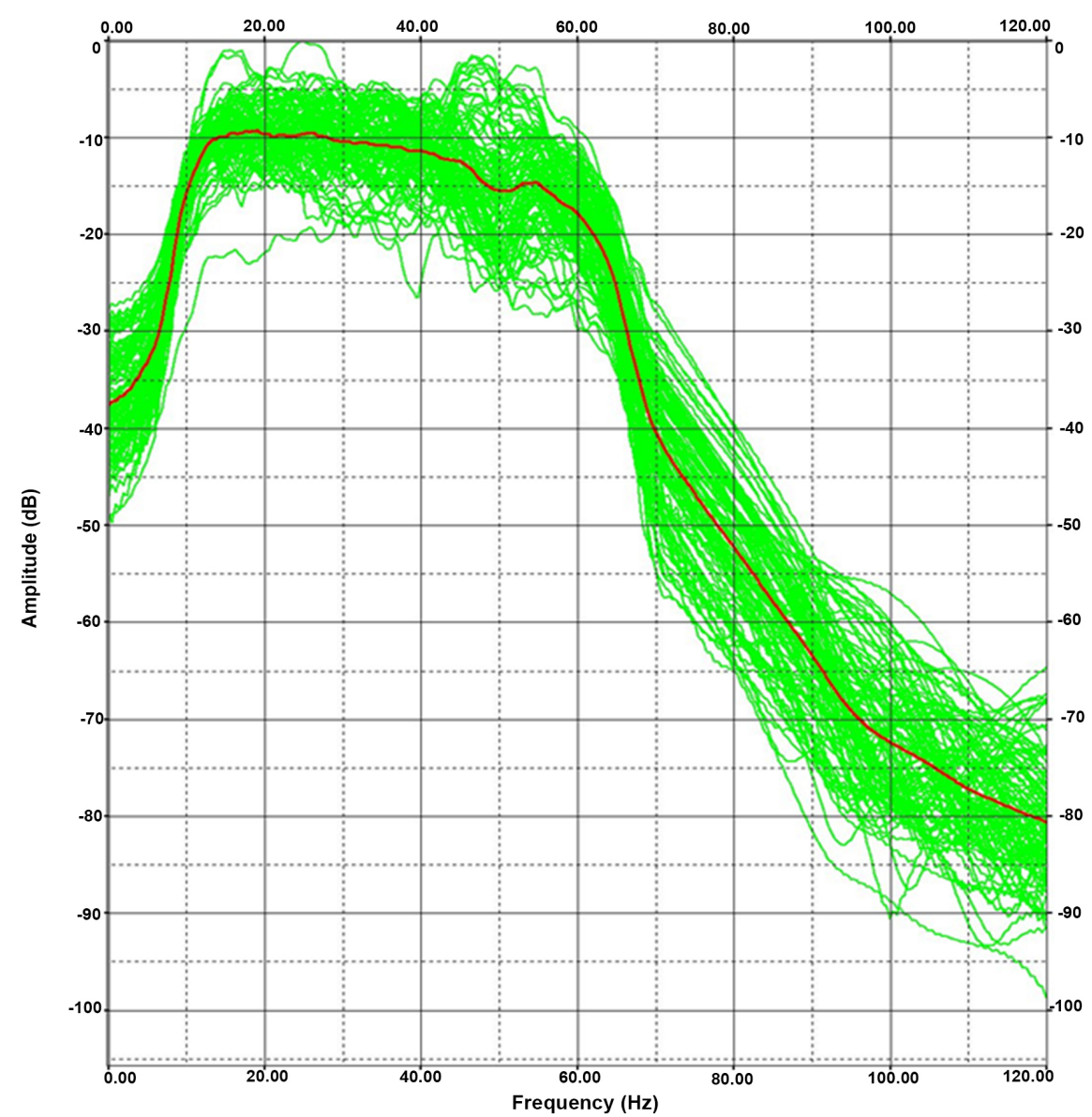

(b)

Figure 6. (a) Shows the shot data with gap deconvolution and gap = $16 \mathrm{~ms}$; (b) Spectrum of band width frequencies with gap deconvolution. 


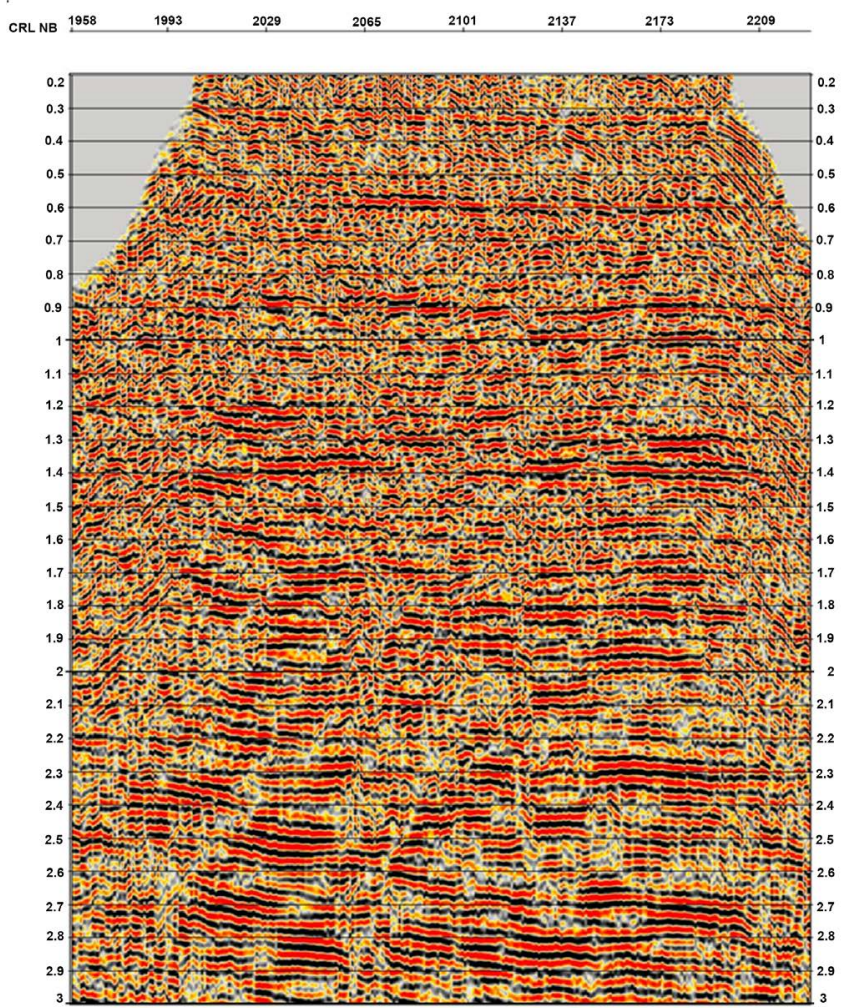

(a)
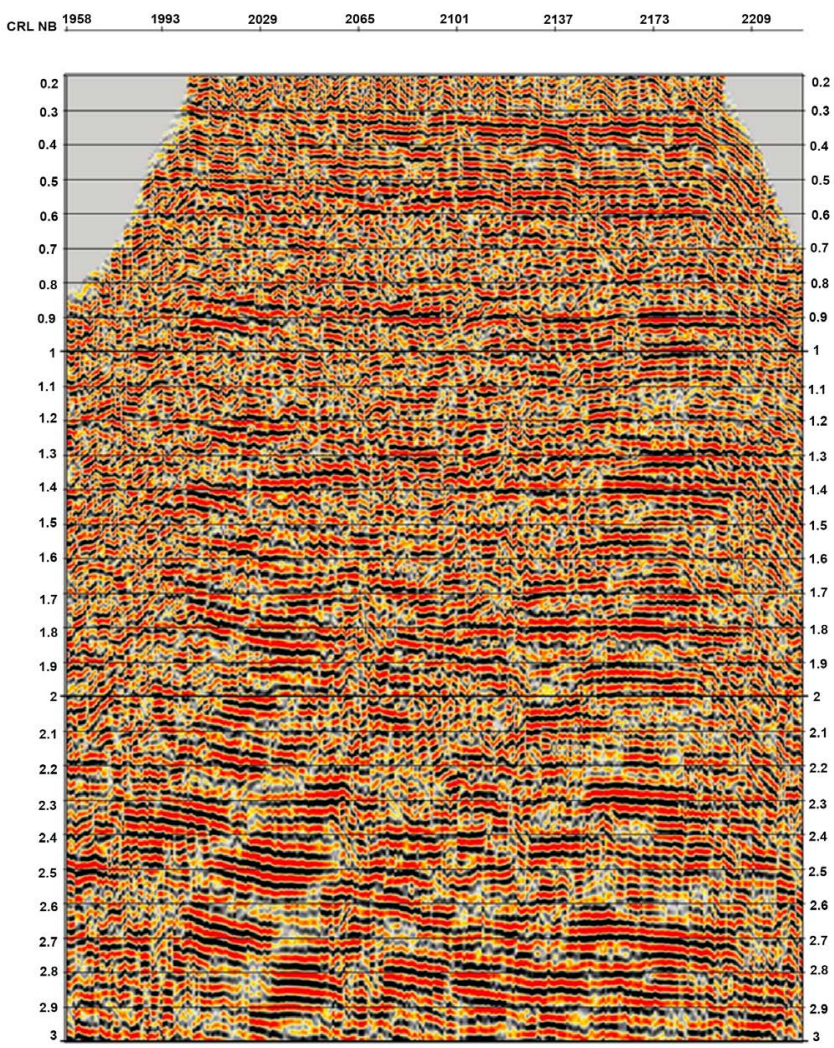

(b)

Figure 7. (a) Shows stack data before deconvolution; (b) Shows stack data after deconvolution. 
in the reflection which correspond to the geology. Such high resolution reflection details are desirable features for seismic interpretation. This paper indicates that suitable parameters can properly enhance the resolution of seismic data.

\section{Conclusion}

The spiking deconvolution operator is the inverse of the (minimum phase) equivalent of the input wavelet. This wavelet may or may not be minimum phase. When the source signature is known, a designature process can be applied as an alternative or a complement to this step in our case, we applied a trace by trace spiking deconvolution, and the deconvolution which in this case desired output (zero lag spike), we used operator length $200 \mathrm{~ms}$. We found the spike deconvolution gives perfect results compared with gap deconvolution because it enhances in the temporal resolution and appears inter layers can be used in the stratigraphy interpretation, but the gap deconvolution gives continuity in reflectors and attenuates in the multiple noise.

\section{References}

[1] Claerbout, J.F. (1976) Fundamentals of Geophysical Data Processing. Blackwell Scientific Publications, Hoboken, 1985.

[2] Leinbach, J. (1995) Wiener Spiking Deconvolution and Minimum-Phase Wavelets. Society of Exploration Geophysicists, London.

[3] De Monvel, J.B., Le Calvez, S. and Ulfendahl, M. (2001) Image Restoration for Confocal Microscopy: Improving the Limits of Deconvolution, with Application to the Visualization of the Mammalian Hearing Organ. Elsevier Academic, Amsterdam.

[4] Drijkoningen, G.G. and Vershuur, D.J. (2003) Seismicdata Processing. Center for Technical Geoscience, Netherlands.

[5] Yalmaz, Ö. (2001) Seismic Data Analysis. Society of Exploration Geophysicists, London.

[6] Robinson, E.A. and Treitel, S. (1980) Geophysical Signal Analysis. Prentice-Hall, Upper Saddle River.

[7] Mhmod, M., Liu, H.Y., Lui, C. and Feng, X. (2016) Performing Spiking and Predictive Deconvolution on 2D Land Data (PSTM). Journal of Geology and Geophysics, $5,239$. 